\title{
Linx
}

Revue des linguistes de l'université Paris X Nanterre

9 | 1997

Émile Benveniste. Vingt ans après

\section{Discours et récit chez les historiens grecs : l'apport des théories de Benveniste}

\section{Guy Lachenaud}

\section{(2) OpenEdition}

\section{Journals}

Édition électronique

URL : http://journals.openedition.org/linx/1040

DOI : 10.4000/linx.1040

ISSN : 2118-9692

Éditeur

Presses universitaires de Paris Nanterre

\section{Édition imprimée}

Date de publication : 1 avril 1997

Pagination : 179-193

ISSN : 0246-8743

\section{Référence électronique}

Guy Lachenaud, «Discours et récit chez les historiens grecs : l'apport des théories de Benveniste», Linx [En ligne], 9 | 1997, mis en ligne le 25 juillet 2012, consulté le 19 avril 2019. URL : http:// journals.openedition.org/linx/1040; DOI : 10.4000/linx.1040 


\title{
Discours et récit chez les historiens grecs : l'apport des théories de Benveniste
}

\author{
Guy Lachenaud
}

$\mathrm{C}^{\prime}$ 'est d'abord à travers le Vocabulaire des institutions indo-européennes que nous avons pu appréhender l'importance de l'oeuvre d'Emile Benveniste, parce que les apports résultant de la comparaison des systèmes linguistiques et notamment du vocabulaire nous permettaient de mieux faire comprendre aux étudiants de Lettres classiques, d'Histoire ou de Sciences Juridiques quelques aspects du sémantisme social. Ce qui nous paraissait caractériser le plus nettement sa méthode et sa pensée, c'était la volonté de ne jamais séparer la description des formes, éclairée par les apports de la grammaire comparée, de leur interprétation. En effet, la corrélation "forme / sens" est nettement établie dans Noms d'agent et noms d'action en indo-européen ou à travers le sémantisme social du Vocabulaire des institutions indo-européennes.

Nous tenons pourtant à préciser d'emblée que ce sont généralement les théoriciens de la littérature, voire les philosophes, qui nous ont incité à relire Benveniste. Depuis longtemps, en dépit des réserves manifestées par certains spécialistes, nous tentons d'approcher les textes des historiens grecs en nous fondant sur les principes suivants : le texte des historiens n'est pas seulement un document objectif dont il importe de vérifier la qualité référentielle et scientifique, il résulte de situations de communication extrêmement complexes (source, code, émetteur, transcodage, décodage, destinataires immédiats ou postérieurs). En préparant des Prolegomènes théoriques à la lecture des historiens grecs et une étude sur les préfaces, nous avons maintes fois rencontré Benveniste, notamment ses considérations concernant l'appareil formel de l'énonciation, les rapports entre discours et récit et cette "subjectivité impliquée, impliquée par l'objectivité attendue" dont parle Paul Ricoeur. Nous citons Ricoeur parce que J.-C. Coquet, dans sa "Note sur Benveniste et la phénoménologie"1 opère un rapprochement entre la théorie de Benveniste

${ }^{1}$ Linx 26,1992, p. 42-48. Cf. P. Ricoeur, Histoire et vérité, Paris, Le Seuil, 1955, rééd. 1964, p. 24. 


\section{Guy Lachenaud}

énoncée dans une page qui concerne l'histoire ${ }^{2}$ et la Sémantique de l'action où Ricoeur définit l'événement, dans les perspectives de la philosophie analytique, comme "un mouvement en tant qu'observable"3. "Les événements sont posés comme ils se sont produits... ils semblent se raconter eux-mêmes", oui, mais parce qu'un je a énoncé préalablement sa propre position. Par ce leitmotiv, Benveniste rejoint aussi bien la phénoménologie que la rhétorique et l'énonciation de la fiction.

Dans un texte fondamental ${ }^{4}$ Benveniste rappelle la distinction saussurienne entre la langue comme "principe de classification" et le langage "multiforme et hétéroclite" qui appartient encore au domaine individuel et social" 5 . Il prolonge ces définitions en énonçant un programme sémiologique large qui inclut la sémiotique, étude des "bases signifiantes de la langue" et reconnaissance des signes, et la sémantique qui prend en charge globalement (et non plus seulement à travers la somme additive des signes) 1 ' "intenté" du discours et nous fait entrer dans le monde de l'énonciation' 6 .

De "l'appareil formel de l'énonciation" nous retiendrons ici deux aspects dont l'importance est cruciale pour la compréhension, dans tous les sens de ce mot, des textes historiques : les jeux avec le temps, ce qui ne surprendra pas le lecteur, mais aussi les relations de personne dans le verbe et dans le récitdiscours 7 .

\section{De la grammaire des pronoms aux relations de personne dans le discours des historiens ${ }^{8}$}

Après avoir cité les grammairiens arabes qui distinguent "celui qui parle", "celui à qui l'on s'adresse" et "celui qui est absent", Benveniste définit la 3ème personne comme la non-personne exclue de la relation fondamentale entre un je, la "personne impliquée", et un $t u$, objet de prédication dans

\footnotetext{
2 P.L.G. I, p. 241.

3 P. Ricoeur, 1977, p. 22.

4 P.L.G. II, chap. 3, "Sémiologie de la langue", p. 43-66 : dans Sémiotica I, La Haye, Mouton \& Co., p. $1-12$ (1969)

5 C.L. G. p. 25.

6 Benveniste, op. cit., p. 64.

7 Cf. "L' appareil formel de l'énonciation" (P.L.G. II, chap. 5, p. 79-88 = Langages 17, Paris, DidierLarousse, 1970, p. 12-18) ; P.L.G I, chap. 18, p. 226-236 : "Structure de relations de personne dans le verbe" = B. S. L 43, 1946, fasc. 1, n 126 et P.L.G. I, chap. 20 : "La nature des pronoms" dans For Roman Jakobson, La Haye, Mouton \& Co., 1956. Cf. aussi P. Ricoeur, "La structure, le mot, l'événement", Esprit 5, 1967, p. 810, 812 et 817, cité par Jean-Claude Coquet, op. cit. p. 43.

8 Le terme de "discours" est évidemment pris ici dans son acception la plus large et désigne toute occurrence d'énoncé (équivalent de la "parole" saussurienne) ou tout groupement de récits et de discours au sens de la théorie de l'énonciation chez Benveniste. Cf. D. Maingueneau, L'analyse du discours, Introduction aux lectures de l'archive, Paris, Hachette, 1991, p. 14-16.
} 
l'énoncé, exclue en somme du dialogue entre deux partenaires. Mais il ne se contente pas d'utiliser les notions grammaticales de personne, de nombre et de diathèse qui structurent le "champ positionnel du sujet", ni de souligner l'ancrage de l'Ego en tant que "centre de l'énonciation"10 et instance de discours à partir de laquelle l'espace discursif s'organise. Quelques remarques lui suffisent pour indiquer que la charge sémantique des pronoms peut dépendre des rapports sociaux : discrétion nécessaire qui conduit à dissimuler le je et le $t u$, formules de politesse, je dilaté ou pluriel dit "de majesté". En outre, chacun des pronoms personnels peut devenir impersonnel et renvoyer à des personnes non-subjectives.

La grammaire des pronoms peut-elle servir à rendre compte du discours des historiens ? Oui, sans doute, mais à condition que l'on considère la théorie de l'énonciation comme devant servir à thématiser la façon dont le sujetnarrateur-historien s'énonce. Ce ne sera guère le cas si l'on se contente de thématiser la façon dont un énoncé a la forme qu'il a ${ }^{11}$.

Il ne nous semble pas artificiel de jeter un pont entre les notations théoriques de Benveniste et les nombreux travaux qui s'efforcent de décliner une typologie des modalités d'énonciation à partir des notions de "point de vue", de "perspective narrative", de "voix", de l'omniscience auctoriale jusqu'au mode dramatique où l'auteur s'efface derrière ses personnages ${ }^{12}$. Dans le cas de l'historien, j'adopterais volontiers le terme d' "observateur-énonciateur" utilisé par J.-C. Coquet, parce qu'il résume bien la tension entre "monde" et "représentation"13. Le monde est ici l'histoire que Benveniste prend bien soin de distinguer de la narration parce qu'elle est aussi en dehors du discours de l'énonciateur-historien et des énonciateurs qui l'informent.

L'histoire permet au locuteur d'avoir des références extérieures à l'énonciation et le locuteur-historien se construit aussi en tant que sujet à travers l'écriture de l'histoire.

\footnotetext{
${ }^{9}$ P.L.G. 1, p. 174 (1950).

10 P.L.G. II, p. 83.

${ }^{11}$ Cf. Sarah de Vogüé, " Culioli après Benveniste", Linx 26, 1992, p. 78-108.

12 Cf. F. Pouillon, Temps et roman, Paris, Gallimard, 1946 ; F. van Rossum-Guyon, Poétique 4, 1970, p. 481 ; N. Friedmann, "Point of View in Fiction: The Developement of a Critical Concept" (1955), dans The Theory of the Novel, éd. P. Stevick, New York, The Free Press, 1967, p. 108-137; M. Bakhtine, La pétique de Dostoïevski, Paris, Seuil, 1970.

13 J.-C. Coquet, op. cit., p. 44. Plat., Rép.III, 392 d sq.
} 
Guy Lachenaud

La personne -Je

(Benveniste: "personne subjective" ou "personne impliquée").

Il est nécessaire, pour analyser le discours des historiens, de distinguer deux foyers de subjectivité, celui qui relève de l'individu inscrit dans une temporalité vécue $(\S 1)$ et l'origine-je du discours, qu'il s'agisse de l'auteur réel ou du narrateur fictif ( $(2)$, auquel il convient d'ajouter toute la gamme des "substituts" du Je (§3).

1) Les préfaces contiennent parfois une signature qui permet de décliner son identité ou son statut social (sphragis, p.e. chez Flavius Josèphe) et quelques éléments autobiographiques qui concernent essentiellement la naissance d'une vocation et sont difficilement séparables de l'énoncé du programme intellectuel de l'auteur (Diodore, Appien, Flavius Josèphe). Ce programme est agrémenté de nombreux topoi qui justifient l'entreprise : la grandeur ou le caractère inédit ou paradoxal du sujet traité, la mémoire et la gloire de l'auteur ou des peuples, le souci d'établir ou de rétablir la vérité et les considérations de méthode, les leçons politiques et morales de l'histoire. Ces éléments paratextuels peuvent être disséminés dans le cours de l'exposé et font parfois l'objet de rappels et de références croisées (Hérodote, Polybe).

Dès les origines du genre historiographique, l'histoire du passé plus ou moins proche (le temps des dieux et celui des hommes chez Hérodote; Xénophon, Cyropédie ) est opposée à celle du présent, ou plutôt d'un passé immédiat dont l'auteur a été le témoin ou même l'acteur (Thucydide; Xénophon, Anabase ). Mais, si cette distinction autorise des controverses épistémologiques et des valorisations qui peuvent s'inverser, elle ne concerne pas la totalité de la pratique discursive. La non-coincidence entre les faits rapportés, l'activité d'observation et d'analyse et la situation d'énonciation fait en effet partie des contraintes du genre : il est impossible d'écrire l'histoire au présent (que peut bien signifier l'expression "présent historique" ?) mais l'enquêteur peut se mettre en scène dans son activité d'enquêteur et faire état de "choses vues" (les monuments sont aussi des erga pour Hérodote), d'archives ou de "traces" (1'"archéologie" de Thucydide). Enfin, quand l'écrivain s'institue donateur de son récit ou projette dans l'avenir son oeuvre et le souvenir de son auteur, le présent et le futur peuvent apparaître.

2) Dans la théorie des genres littéraires esquissée par la Poétique d'Aristote, l'histoire, en tant qu'exposé détaillé des faits qui se sont produits (kath' hekasta ), apparaît comme un contre-exemple, dans la mesure où Aristote privilégie le drame comme exemple de muthos et de mimésis. Pourtant, le Stagirite n'ignore pas la filiation entre l'épopée et l'histoire : l'activité narrative est bien pour lui une catégorie englobant ce que l'anglais appelle story et history. La "mise en intrigue", 1"agencement des faits en système" (50 a 5 : è tôn 
pragmatôn sustasis ) caractérisent aussi le discours des historiens au niveau sémantique, en tant qu'ensemble de phrases ${ }^{14}$.

Ce n'est pas derrière ses personnages que l'historien devrait s'effacer, comme Homère ou les Tragiques, mais derrière les faits, afin de produire ce que Platon appelle un récit "simple", un discours qui ne serait même pas assertif ("les faits se sont bien déroulés ainsi"). Tour à tour, et souvent pour un même ensemble de faits, Hérodote se comporte en narrateur omniscient qui cède à la joie homérique de raconter, aligne les versions contradictoires et tente de réduire l' "hétérogénéité énonciative" et la discordance qui proviennent de ses informateurs, construisant ainsi à l'intérieur de son propre discours des niveaux distincts grâce à des paraphrases, gloses, incidentes, parenthèses ou jugements que des embrayeurs permettent de repérer ${ }^{15}$.

3) Parmi les substituts du Je, il convient de distinguer tout d'abord les possibilités offertes par la grammaire des pronoms, le Nous dit de majesté qui correspond à un "Je dilaté", comme dit Benveniste (assez fréquent chez Hérodote), le Nous "inclusif" = Moi + Vous (p.e. le peuple juif pour Flavius Josèphe). Mais d'autres substituts sont plus intéressants pour notre propos. Dans les hymnes préliminaires des poèmes épiques et didactiques, la Muse inspiratrice se substitue au poète ${ }^{16}$. Dans les Enquêtes d'Hérodote, nous trouvons des instances narratives déléguées, dénommées ou anonymes, individuelles ou collectives : les récits rapportés sont soit liés à la trame par des liens très forts ${ }^{17}$ soit insérés ou enchâssés quand il s'agit d'exempla ou d'anecdotes à caractère symbolique ${ }^{18}$.

La "personne non-je" (Tu ou Vous ) : "la personne non-subjective", la personne interpellée ou la "personne qu'on se représente".

Contrairement à ce qui se passe dans les poèmes épiques, didactiques ou philosophiques, le donateur ou l'inspirateur (la Muse pour Homère) ou le dédicataire ne sont pas présents expressément dans les oeuvres historiques de l'époque classique, mais leur présence est parfois manifeste dans les oeuvres plus tardives (Epaphrodite pour Flavius Josèphe, Constantin pour Eusèbe, Trajan pour Pline le Jeune). Ces personnages se confondent parfois avec l'inspirateur, le dédicataire ou le héros de l'oeuvre. Le grec, comme d'autres langues, offre la possibilité de recourir à un $t u$ fictif, une "quasipersonne", qui

\footnotetext{
14 Cf. P. Ricoeur, Temps et récit, t. 1, p. 60, note 2 (remarques sur les traductions proposées par les derniers éditeurs de la Poétique, R. Dupont-Roc et J. Lallot, Paris, Seuil, 1980) et La métaphore vive, Paris, Seuil, 1975, p. 88- 100, qui se réfère expressément à Benveniste.

15 D. Mainguencau, op. cit., p. 145-146 (métadiscours et paraphrases, ironie).

16 Cf. infra, p. 10 sq.

17 Cf. VIII, 35, infra, p. 7.

18 Cf. p.e. VI, 86 ou VIII, 104-106.
} 


\section{Guy Lachenaud}

correspond au on du français. Le(s) destinataire(s) de l'oeuvre peut être présent sous les formes déguisées de la non-personne.

\section{"Il(s) : "la non-personne", "forme non-personnelle de la flexion verbale".}

Nous pouvons distinguer les autres, dénommés ou anonymes, exclus de la relation "je-tu" par leur éloignement dans le passé, ou volontairement exclus de cette relation dans le présent. Dans le premier cas, il s'agit de la tradition écrite et orale (l'akoé de Thucydide ou les logioi du proème d'Hérodote), des écrivains prédécesseurs (discours indifférent, objectif, laudateur, critique ou polémique) ou encore des ancêtres. Dans le second cas, l'usage de la nonpersonne peut correspondre aux sentiments d'hostilité ou de mépris (les Romains ou les Grecs pour Flavius Josèphe) ou concerner les destinataires de l'oeuvre (les pepaideumenoi pour Strabon, les hommes soucieux de comprendre le passé et la raison des événements pour Thucydide, Xénophon et Polybe).

La non-personne, ce sont aussi les hommes en général, dans le commentaire qui accompagne le récit et dans les sentences ou les développements de nature morale, intellectuelle ou politique. Thucydide se réfère par exemple au "caractère humain" des événements (to anthropeion), ou à ce que l'on est bien obligé de traduire par "nature humaine". Enfin, les personnages de l'oeuvre, personnes réelles en principe, c'est-à-dire historiques, sont parfois mythiques ou fictifs, dans certains récits insérés ou enchâssés, et l'on se souvient de la définition aristotélicienne de la fiction comme "mimésis d'agissants". La pratique des discours reconstitués, sujette à caution puisque Thucydide éprouve le besoin de se justifier en 1, 22 dans sa seconde préface, et l'utilisation des personnages comme truchement pour l'expression des idées personnelles ou l'élucidation de l'événement vont dans le même sens.

\section{Récit et discours : "les jeux avec le temps"}

Platon est souvent cité comme le premier théoricien qui ait affirmé la pluralité et le feuilletage des textes diégétiques ${ }^{19}$. Le récit, en tant que mimésis praxeôs et logique des actions figurées, peut-il être simplement diégétique, comme Platon l'envisageait à travers son travail de réécriture du texte homérique ? Selon H. Weinlich, "en histoire, la structure fondamentale de la représentation consiste à enchâsser du récit dans le commentaire"20. Il serait sans aucun doute nécessaire, du point de vue de l'histoire du genre littéraire de l'historiographie, de faire varier la relation entre les termes "récit" et "commentaire", y compris quand il s'agit de quasi-contemporains comme Hérodote et Thucydide (20 ans de différence). A noter que Ricoeur hésite à

\footnotetext{
19 Plat., Rép. III, 392 d sq.

${ }^{20}$ H. Weinrich, Tempus : Besprochene und erzâhlte Zeit, Stuttgart, Verlag W. Kohlhammer, 1964, trad. fr. de M. Lacoste, Le Temps. Le récit et le commentaire, Paris, Seuil, 1973, p. 79.
} 
adopter le terme de "commentaire", parce qu'il n'implique pas suffisamment la "tension" du débat et les conflits multiples que font naître les interprétations des événements ${ }^{21}$.

La verbalisation, la description et surtout la mise en intrigue introduisent de nouvelles configurations et le discours accompagne plus ou moins la diégésis, au point de devenir discours affectif ou idéologique. Les titres de Genette, "Frontières du récit" et "Le discours du récit", des expressions comme "récit d'événements" et "récit de paroles" et l'assimilation du discours à des expansions du récit traduisent bien les apories du discours théorique ${ }^{22}$. La rhétorique classique, qui met en jeu des notions comme copia rerum, inventio ou dispositio, ou bien encore docere, placere, movere (Cicéron), établit déjà la distance entre la matière informelle (cf. la métaphore de la forêt ou de $l^{\prime}$ amethodos hylé utilisée par les anciens et reprise par les humanistes comme Guillaume Budé), le travail littéraire d'adaptation et de sélection qui prend en compte les situations de communication ou l'intérêt présumé du lecteur et les modalisations axiologiques qui tour à tour découlent du récit et le rendent licite.

Malgré les contraintes particulières qui pèsent sur le genre historiographique, les "jeux avec le temps" qui caractérisent la poétique narrative s'y manifestent également, à trois niveaux, celui de la temporalité vécue, celui du système paradigmatique des temps du verbe (Ricoeur ${ }^{23}:$ lle moyen de moduler temporellement tous les verbes d'action le long de la chaîne narrative"), celui de la disposition syntagmatique.

Les choses sont apparemment très claires: selon Benveniste, le passé n'implique pas la sui-référence du locuteur à sa propre énonciation, comme dans le discours : "pour qu'ils puissent être enregistrés comme s'étant produits, ces faits doivent appartenir au passé" (ce qui s'apparente à une lapalissade!). C'est pourquoi il ajoute: "Sans doute vaudrait-il mieux dire : dès lors qu'ils sont enregistrés et énoncés dans une expression temporelle historique, ils se trouvent caractérisés comme passés"24. C'est en principe le rôle essentiel de l'aoriste, bien que ce ne soit pas le seul (cf. le texte de Thucydide étudié cidessous). Il se peut d'ailleurs, selon le mot de Roland Barthes ${ }^{25}$, que l'emploi du passé simple connote davantage la littérarité du récit qu'il ne dénote le passé de l'action et il faudrait ici faire intervenir l'ensemble des modalisations aspectuelles, ce qui nous entraînerait trop loin: accompli-non accompli, duratif-instantané...

21 P. Ricoeur, Temps et récit, $t$. II, p. 101.

22 G. Genette, "Frontières du récit", Figures II, Paris, Seuil, 1969, p. 49-69, et "Le discours du récit", Figures III, Paris, 1972, p. 65-273.

23 P. Ricoeur, Temps et récit, t. II, p. 93.

24 Benveniste, p. 239. Le texte fondamental est l'article de 1965, "Le langage et l'expérience humaine" (P.L.G II, notamment p. 68, 73).

${ }^{25}$ R. Barthes, Le degré zéro de l'écriture, Paris, Le Seuil, 1953 (cf. G. Genette, Nouveau discours du récit, Paris, Le Seuil, 1983, p. 53). 


\section{Guy Lachenaud}

Les successeurs de Benveniste se sont plus intéressés au rôle du discours dans le récit qu'à la distinction formellement établie par Benveniste entre discours (toute énonciation rapportée à sa situation d'énonciation) et récit qui efface les marques de la présence de l'énonciateur. L'absence du narrateur est désormais analysée comme une stratégie.

\section{Deux exemples de récit}

Hérodote, VIII, 35-36: "Le raid sur Delphes"

Thucydide, III, 81-83 : "La guerre civile à Corcyre"

Les littératures grecque et latine présentent beaucoup de récits parallèles qui permettent de repérer des stratégies narratives différentes et la réinterprétation des traditions (par exemple Hérodote et Plutarque). Mais nous avons choisi de nous en tenir à Hérodote et Thucydide. Il est d'usage, depuis Cicéron, d'opposer la fluidité narrative qui caractérise Hérodote, le plus homérique de tous les historiens, et les aspérités du discours thucydidéen qui en rendent difficile la lecture. Du côté d'Hérodote, la souplesse mimétique et sympathique, du côté de Thucydide, un intense travail cérébral et l'intrusion perpétuelle de l'intelligence.

\section{Hérodote :}

Le découpage du texte :

VIII, 35 : S'ils suivaient ce chemin, s'étant séparés du reste de l'armée, c'était afin_de piller le sanctuaire de Delphes et d'en présenter au Roi les trésors ; Xerxès, (selon mes informations), connaissait, d'après tant de récits qu'on en faisait sans cesse,...

VIII, 36: A cette nouvelle, les Delphiens furent saisis d'une grande épouvante et, pleins de frayeur, ils consultaient l'oracle au sujet des trésors sacrés pour savoir s'ils devaient les enfouir en terre ou les transporter dans un autre pays; mais le dieu leur interdisait de les déplacer, déclarant qu'il était bien capable à lui seul de défendre ce qui était à lui. Après avoir entendu cette réponse, les Delphiens se préoccupèrent de leurs propres affaires ;...

VIII, 37: Les Barbares en marche approchaient, ils étaient en vue du sanctuaire et c'est alors que le prophète, dont le nom était Akératos, voit disposées en avant du temple, transportées là de l'intérieur hors de la grande salle, les armes, armes sacrées auxquelles aucun homme n'avait le droit de toucher... ils se trouvent face à des prodiges encore plus frappants que le prodige précédent. Sans doute en effet, c'est déjà chose très surprenante qu'on ait $p u$ voir des armes de guerre transportées d'elles-mêmes hors du temple devant la façade; mais ce qui se produisit ensuite mérite au plus haut point entre les phénomènes merveilleux de provoquer l'étonnement. 
VIII, 39: Ceux d'entre eux qui revinrent de cette expédition racontaient, selon mes informations, qu'outre les prodiges dont j'ai parlé, ils avaient vu d'autres manifestations divines: deux hoplites d'une taille plus qu'humaine les poursuivaient, les massacrant et leur donnaient la chasse ; ces deux guerriers, au dire des Delphiens, étaient des héros locaux, Phylakos et Autonoos... Les blocs de pierre tombés du Parnasse étaient encore intacts de nos jours, gisant dans l'enclos d'Athéna Pronaia, là où ils s'abattirent en roulant à travers les Barbares. Voilà dans quelles conditions ces hommes s'éloignèrent du sanctuaire.

1) L'occasion et la nature du récit : Hérodote prend bien soin, en utilisant ce qu'un commenteur appelle "framing sentences", d'insérer l'épisode dans la série des lieux traversés par l'envahisseur. La première phrase situe l'action et énonce les intentions des Barbares (je passe sur les commentaires historiques suscités par cet énoncé qui laisse les érudits perplexes). Il s'agit, en effet, d'une hypothesis transmise, sinon par la tradition, du moins par les Delphiens, dont certains ont été témoins : le récit correspond à l'horizon d'attente du lecteur, il n'est pas un pur artefact.

2) Bien que l'historien se retranche derrière deux pluriels anonymes (ses informateurs, Delphiens ou Perses), les connecteurs logiques de la démonstration $(\gamma \dot{\alpha} \rho)$ lui appartiennent et le lecteur est contraint de reconnaître, qu'aux yeux d'Hérodote, le prodige des blocs de pierre est plus prodigieux que le déplacement spontané des armes sacrées, ce qui est sans doute contraire à son attente.

3) Les intrusions passagères de l'enquêteur ( $\omega \dot{s} \dot{\varepsilon} \gamma \dot{\omega} \pi v v \theta \dot{\alpha} v o \mu \alpha l)$ :

- sans désignation des informateurs qui peuvent être des Asiatiques plutôt que des Delphiens (à propos de la connaissance qu'avait Xerxès des trésors de Delphes).

- une remarque concernant les armes sacrées

- la mention des informateurs barbares vient corroborer l'apologétique delphique

- indications topographiques et choses vues par l'enquêteur.

Catherine Darbo-Peschanski ${ }^{26}$ a-t-elle raison de considérer que Hérodote ne fait que prêter la parole à ses informateurs et "se pose en propriétaire de son texte" ? Elle dit elle-même qu'il lui arrive de déléguer la parole à des "instances narratives floues" 27 ou, ce qui est bien plus fréquent, à "des informateurs désignés" ou anonymes. "Ces récits qui ne sont attribués à personne et dont aucune marque ne vient signaler l'altérité, se signalent seulement par leur homogénéité et la clôture parfaite de l'anecdote qui les isolent du reste du texte. Ils sont régis par la seule logique narrative $e^{28}$. A coup sûr, cette présentation ne convient pas en tout point à notre texte : s'il y a clôture et altérité, il y a aussi

\footnotetext{
${ }^{26}$ C. Darbo-Peschanski, Le discours du particulier. Essai sur l'enquête hérodotéenne, Paris, Le Seuil, p. 108.

27 Usage du "nous" par exemple en II, 148 ou VII, 214.

28 Ibid. p. 124.
} 


\section{Guy Lachenaud}

confrontation des informations et utilisation d'une démarche de type sémiologique (les blocs de pierre).

3) L'enquêteur qualifie son récit de prodigieux en anticipant sur la narration des prodiges. La mise en condition du lecteur, à la fois par les effets de réel et par le commentaire, est assurée de réussir parce qu'elle rencontre la croyance et la tradition, mais elle s'accompagne d'une certaine mise à distance, puisque le rapport entre la réponse oraculaire rapportée en style indirect et le récit a moins de valeur probatoire en fin de compte que la foudre. L'opposition

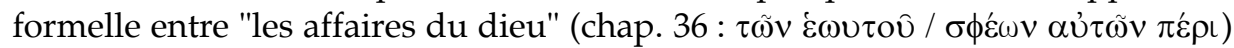
constitue un indice supplémentaire et sans doute une marque d'ironie.

4) L'expression de la temporalité : tempo et scénographie. Au début du chapitre 36, les aoristes concernent la série des décisions et des actions tandis

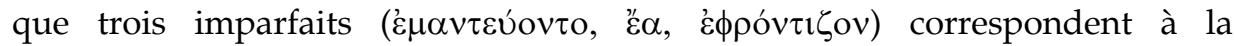
consultation oraculaire et à la perplexité qu'elle entraîne. Une telle variation est difficilement traduisible. Au chapitre 37, deux présents, que l'on pourrait appeler "présents d'ostension" plutôt que "présents historiques", correspondent à l'irruption des prodiges.

\section{Thucydide}

\section{Le découpage du texte :}

81, 4 : Pendant les sept jours qui suivirent l'arrivée d'Eurymédon et où il fut là avec ses soixante navires, les Corcyréens firent un carnage de leurs concitoyens qui passaient pour opposants ; ils en rejetaient la responsabilité sur les adversaires de la démocratie, mais certains moururent aussi victimes de haines privées, et d'autres, qui avaient prêté de l'argent, sous les coups de leurs débiteurs.

81, 5: La mort revêtit toutes les formes et, (comme cela se produit en pareil cas,) on ne recula devant rien, - et pis encore. Le père tuait son fils, les suppliants étaient arrachés des sanctuaires ou tués sur place, certains périrent même emmurés dans le sanctuaire de Dionysos.

$821:$ Tel fut en effet le degré de cruauté qu'atteignit la guerre civile, et il fut plus sensible parce qu'elle était des toutes premières; car ensuite, le mouvement gagna pour ainsi dire le monde grec tout entier... $2 \mathrm{~A}$ la faveur des troubles, on vit s'abattre sur les cités bien des maux, comme il s'en produit et s'en produira toujours tant que la nature humaine restera la même, mais qui s'accroissent ou s'apaisent et changent de forme selon chaque variation qui intervient dans les conjonctures. En temps de paix et de prospérité en effet, les cités et les particuliers ont un esprit meilleur...

82, 3 : Ainsi la guerre civile régnait dans les cités,... 4 On changea jusqu'au sens usuel des mots par rapport aux actes, dans les justifications qu'on donnait.

82, 8: La cause de tout cela, c'était le pouvoir voulu par cupidité et par ambition : de ces deux sentiments provenait, quand les rivalités s'instauraient, une ardeur passionnée... 
83, 1: C'est ainsi que la dépravation revêtit toutes les formes dans le monde grec par l'action de la guerre civile, et la simplicité, où la noblesse a tant de part, disparut sous les railleries, tandis que l'affrontement d'esprits défiants passa au premier plan :

1) Les massacres de Corcyre ne sont qu'un prétexte et fournissent l'occasion d'amorcer le thème des transformations induites par la guerre, de ses effets sur la nature humaine. Le début de la guerre et les horreurs de la guerre civile méritaient sans doute une narration pathétique, mais, même quand il s'agit de la peste d'Athènes, de la prise de Platées et en général des récits de bataille, le discours qui manifeste l'exigence de clarté, mais aussi la propension à disserter, à analyser le comportement des hommes, l'emportent constamment sur la description ou la narration au sens strict. Certains commentateurs relèvent même, par exemple dans les récits de bataille, le déséquilibre entre les logoi reconstitués des acteurs et les erga au point que ceux-ci ne sont plus qu'un pâle reflet ${ }^{29}$. Mais ici, ce n'est même pas le cas, puisque les éléments diégétiques et discursifs sont difficilement repérables (les temps des verbes peuvent servir d'indices, mais il est exclu de trouver des "présents historiques").

2) Bien que des indications temporelles insèrent les sept journées de massacres dans la trame du récit $(81,4)$, Thucydide se soucie plus de dégager la signification générale de l'épisode que de le mettre en scène. Dans le début du texte, le jeu des aoristes et des imparfaits ne fournit pas d'indications claires sur la répartition entre récit et discours. L'aoriste est tantôt de type narratif ou historique (ponctuel ou inchoatif : événement ou début d'un processus), tantôt récapitulatif, par exemple en 81,4 et 83,1 , dans une formule reprise sous forme anaphorique que l'on peut comparer aux procédés archaïques de la

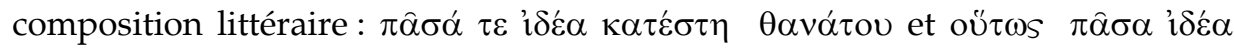

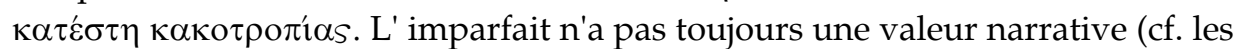
verbes d'action en 81,5 ), il sert plus souvent à la description des sentiments et des pensées. Peut-on dire que l'aoriste met en relief dans l'ordre du récit, tandis que l'imparfait signale le glissement à l'arrière-plan des contenus racontés (cf. Weinrich) ? En tout cas, il y a des variations de tempo.

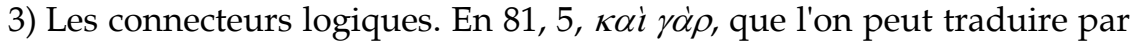
"car aussi bien", introduit des éléments de récit qui justifient la généralisation qui précède. En 82,3 , oủv introduit un passage descriptif à l'appui d'une généralisation énoncée au présent. On peut aussi noter l'abondance des connexions logiques dans la description morale $(82,8)$.

Le texte de Thucydide, dans sa structure double et déséquilibrée, illustre fort bien la distinction kantienne rappelée par Ricoeur entre le jugement déterminant qui s'investit totalement dans l'objectivité qu'il produit et le jugement réfléchissant ${ }^{30}$.

${ }^{29}$ Cf. V. Hunter, Thucydides, The Artful Reporter, Toronto, 1973.

30 Ricoeur, Temps et récit, t. II, p. 92. 
Guy Lachenaud

\section{L'exemple des poèmes, préfaces ou incipit}

Bien que le début des oeuvres soit souvent un paratexte surajouté ou une proclamation que la suite de l'oeuvre peut démentir ou infléchir, il peut contenir des indices qui caractérisent les rapports du Sujet-Narrateur avec le discours qu'il énonce et par lequel il s'énonce. Ainsi, dans une perspective qui se veut à la fois sémiotique et anthropologique, Claude Calame, lecteur des historiens, mais surtout des poètes, tente, dans un chapitre intitulé "Enonciations", de poser en termes nouveaux la question du sujet qu'il se refuse à enfermer dans "la trame des structures sociales et la trame des structures de l'inconscient" et remarque que, dans les poèmes homériques, le "Je-Producteur" se projette dans un Tu dont il n'est que le porte-parole (les Muses). En disant cela, il ne fait que déconstruire l'appareil formel de l'énoncé et ne dit rien du sujet et de son rapport aux Muses en tant qu'objet de culte et de croyance.

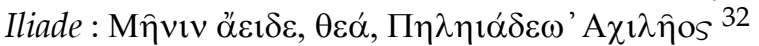

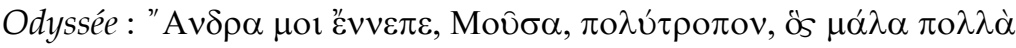

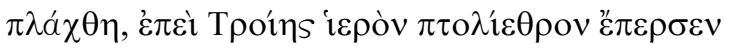

Les formules qui introduisent les Hymnes homériques sont analogues:

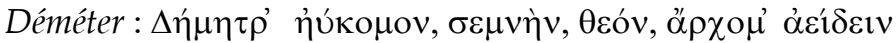

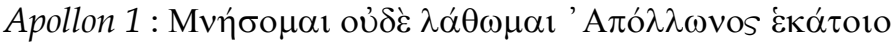

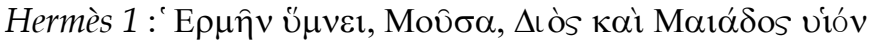

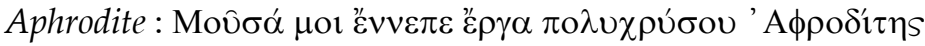

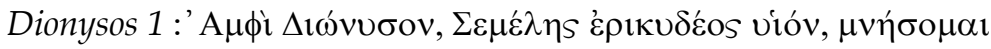

La distribution des éléments dans ces incipit fait apparaitre qu'il n'y a pas d'ordre canonique :

- le début de l'Odyssée inverse purement et simplement le schéma de la communication tel que le présente Jakobson et présente la séquence suivante : objet = oeuvre (accusatif), destinataire (au datif), source, garant, donateur (vocatif-nominatif) comme au début d'Hermès I. L'Iliade omet même d'exprimer la personne du destinataire.

- mais les Hymnes manifestent déjà (si l'on admet que la composition des Hymnes est postérieure à celle des poèmes cycliques) des signes d'appropriation de l'oeuvre par l'aède. Il devient l'actant essentiel, sujet d'un verbe au présent, au futur et même au subjonctif, désignant le début du chant (Déméter 1), l'acte de mémoire ou de chant (Dionysos 1, Apollon). Le Narrateur-

\footnotetext{
31 C. Calame, Le récit en Grèce ancienne, Méridiens, Klincksieck, 1986, p. 11.

32 M. Croiset note dans une édition scolaire : "La Muse (appelée ici simplement "déesse", $\theta \varepsilon \alpha ́$ ) est censée chanter par la bouche de l'aède, puisque celui-ci ne fait qu'énoncer ce qu'elle lui dicte. Du reste, les aèdes ne chantaient pas, à proprement parler..." Le lecteur appréciera l'efficacité de ces considérations positivistes : l'énoncé n'est analysé qu'en tant que reflet des réalités sociales et culturelles.
} 
Hésiode, dans le début de la Théogonie, reste dépendant des Muses pour l'inspiration, bien qu'il affirme l'autonomie de son activité artisanale de poète.

Après Homère, il se produit un élargissement du "Je-Tu-Nous" vers un "Ils", collectif limité qui fait affluer les informations vers la source de l'énonciation. Chez Hérodote, le garant de l'énoncé est une troisième personne du pluriel, les informateurs : "avec Hérodote apparaît le discours historique à la troisième personne" et l'utilisation du document (écrit ou oral) limite les interventions $\mathrm{du} \mathrm{Je} \mathrm{J}^{33}$.

\section{Hérodote}

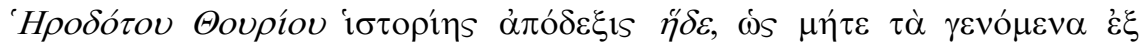

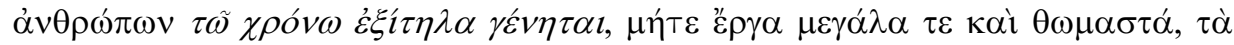

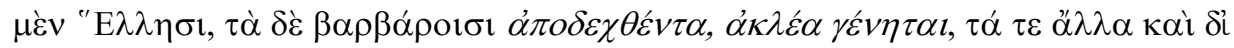

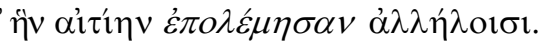

" $D$ 'Hérodote de Thourioi voici le compte-rendu d'enquête afin que les actes qui furent ceux des hommes ne soient pas abolis par le temps et que les exploits considérables et prodigieux accomplis tant par les Grecs que par les Barbares ne restent pas sans renom, et en particulier (l'exposé) des raisons pour lesquelles ils se firent la guerre les uns aux autres".

Cette phrase nominale contient d'abord une signature et l'équivalent d'un titre (le génitif a la double valeur de complément d'agent et de complément de nom), suivi d'un indice d'ostension et d'offrande ( $\not \delta \varepsilon$ : démonstratif de première personne accordé, comme le veut la grammaire, avec le féminin $\alpha \dot{\alpha} \delta$ ó $\delta \xi 1 s)$, avant d'instaurer un dédoublement entre l'enquête et l'exposition (au double sens de ce mot) des résultats de l'enquête. Hérodote énonce ensuite les intentions de son discours en renvoyant, comme le poète

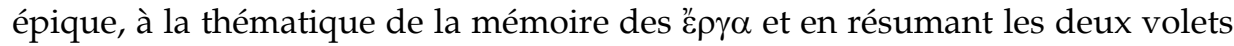
$\mathrm{du}$ diptyque, le thème large et le thème restreint. Même en l'absence de la Muse, dont le mythe nous dit qu'elle est fille de Mnémosyne, il est clair, qu'en présentant son logos comme l'exposé d'une enquête, Hérodote feint de s' effacer derrière les erga et ses informateurs.

Examinons maintenant le début du logos de Cyrus :

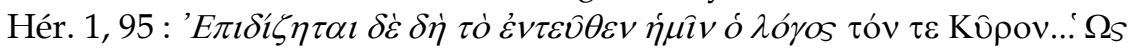

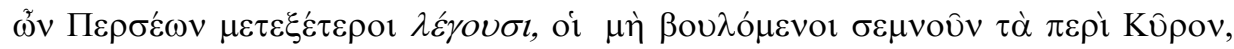

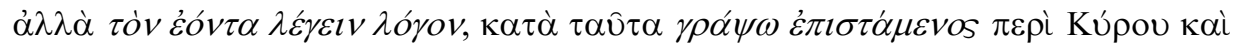

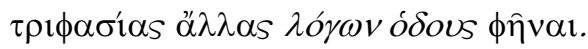

33 Les traducteurs rencontrent généralement de grandes difficultés pour rendre compte de l'énonciation. Parce que nous estimons qu'il est nécessaire d'être plus rigoureux, nous proposons des traductions personnelles. 


\section{Guy Lachenaud}

"A partir de maintenant notre récit se met en quête de savoir qui était Cyrus... Eh bien, ce qu'en disent certains des Perses, ceux qui ne veulent pas magnifier l'histoire de Cyrus mais énoncer la réalité, je $m^{\prime} y$ conforme en écrivant, bien que je sache aussi trois voies différentes pour parler de Cyrus".

L'usage du mot $\lambda$ ó $\gamma$ os en tant que sujet grammatical d'un verbe qui signifie "rechercher" est intéressant, mais difficile à interpréter : il y a, si l'on peut dire, une logique de la diachronie historique et narrative et des contenus narratifs qui s'impose à l' historien, tout comme à l'aède qui doit suivre la "route"34. Ailleurs, Hérodote fait état des contraintes du logos qui pèse sur son logos, sans que l'on puisse déterminer nettement s'il s'agit de contraintes externes provenant des contenus narratifs et de la diachronie historique, ou d'exigences de rationalité et de justice qui lui sont propres ou qu'il fait siennes $^{35}$. Dès lors que sont en jeu l'abondance de l'information et la nécessaire sélectivité et plus encore le rapport du discours à l'être, le sujet de conscience

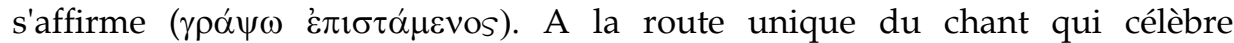
s'opposent les voies (les voix) multiples.

\section{Thucydide}

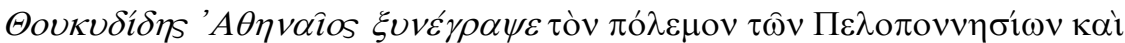

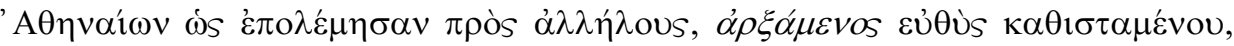

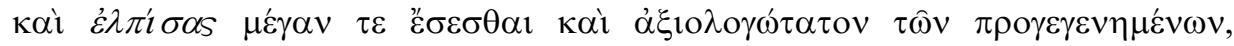

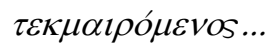

"Thucydide l'Athénien a composé le récit de la guerre que se firent les Péloponnésiens et les Athéniens: il avait entrepris ce travail dès qu'elle commença et avait prévu que le conflit serait important et mériterait plus que tous les précédents d'être raconté ; il faisait cette conjecture... "

Le contraste avec l'incipit d'Hérodote est radical puisque le nom de Thucydide est sujet d'un verbe à l'aoriste qui désigne l'activité de composition. Bien que l'oeuvre soit qualifiée plus tard, dans une formule célèbre, de "trésor pour toujours", elle n'est pas présentée ici comme un produit fini : Thucydide transporte son lecteur à l'instant où la guerre a éclaté et déclenché en lui cette activité de prévision qu'il s'arroge souverainement. Les deux temporalités, biographique et événementielle, coïncident en effet et les participes apposés

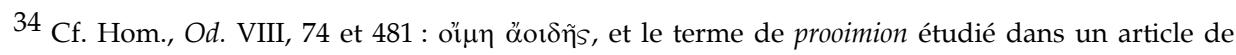
J. Lallot et R. Dupont-Roc, "Le $\pi \rho o_{1} \mu 1$ ov est-il un proème ?", dans Le texte et ses représentations (Etudes de littérature ancienne 3, Presses de l'ENS, Paris, 1987, p. 13-27.

35 Ce dédoublement du logos est très présent dans la philosophie d'Héraclite (cf. les commentaires de M. Conche, Héraclite, Fragments, Paris, PUF, coll. Epiméthée, 1986, p. 23 sqq.) Cf. l'anatiké logographiké dont Platon parle dans le Phèdre $264 \mathrm{~b}$ et G. Lachenaud, Mythologies, religion el philosophie de l'histoire dans Hérodote, Atelier de reproduction des thèses, Univ. de Lille, 1978, p. 69-71. 
correspondent à des opérations intellectuelles. Chez Thucydide, l'omniscience s'énonce aussi sur le mode de la prospective : capacité de prévision du témoin des événements, mais aussi perception des enchaînements qui se prolongeront et spéculation sur les rapports entre événements et nature humaine. Nous sommes bien loin de la prolepsis proprement narrative à laquelle recourt le narrateur omniscient quand il annonce ce que l'on va lire à travers des discours de type prédictif (divination, avertissement, conseil, délibération) ou en produisant des éléments narratifs multipliés qu'il délègue à des énonciateurs différents.

\section{Conclusion}

Les considérations épistémologiques de Benveniste devraient à notre sens lui éviter d'encourir le reproche de s' être enfermé dans la clôture du signe, si tant est que cette présentation caricaturale de la linguistique structurale soit pertinente. Comment désapprouver son souci constant de tenir compte des possibilités offertes par la langue sous tous ses aspects, y compris la syntaxe? Après tout, ce souci devrait être partagé par tous ceux qui réhabilitent l'explication de texte, pratiquent l'analyse de discours ou étudient la poétique des oeuvres particulières, l'art et la manière des écrivains. Cependant, même chez des lecteurs aussi bien disposés que Ricoeur, nous percevons les effets du post-structuralisme : critique du binarisme saussurien, retour d'une philosophie du sujet, même s'il s'agit d'un sujet clivé ou divisé, immergé dans l'univers des représentations qui lui viennent d'ailleurs, comme l'est nécessairement l'historien, plaidoyers pour le récit. Prolonger, mais aussi dépasser Benveniste, tel semble être le mot d'ordre. Bien qu'il ait jeté les bases d'une théorie de l'énonciation, nous avons dit qu'il était nécessaire d'affiner sa théorie des rapports entre discours et récit. Il suffira ici d'évoquer les théories de la focalisation et les développements de la narratologie, notamment les définitions proposées par Mieke Bal qui dépassent le binarisme et proposent une division hiérarchique et ternaire : le texte narratif est un "texte dans lequel une instance raconte un récit", le récit est le "signifié d'un texte narratif", l'histoire est une "série d'événements logiquement reliés entre eux, et causés ou subis par des acteurs" 36 . Une narratologie ne peut contribuer utilement à la lecture que si elle s'abstient d'isoler ces différents registres.

\footnotetext{
${ }^{36} \mathrm{M}$. Bal, Narratologie (Essais sur la signification narrative dans quatre romans modernes). Hes Publishers, Utrecht, 1984, p. 4 sq. Cette division hiérarchique et ternaire se retrouve, avec des différences, chez R. Barthes ("Introduction à l'analyse structurale des récits", Comm. 8, 1966), L. Dolezel ("Motif Analysis and the System of Sensitivity in L'étranger", dans P. Léon et al., Problèmes de l'analyse textuelle, Montréal-Paris-Bruxelles, 1971) et G. Genette (introd. au "Discours du récit", Figures III, Paris, Seuil, 1972). Selon Barthes, l'analyse descriptive se fait à trois niveaux, celui des fonctions (cf. Propp), celui des actions (cf. Greimas), celui de la narration. Dolezel distingue quatre 'blocks" : l'histoire, les personnages, l'espace et les interprétations. Selon Genette, la narration concerne le procès d'énonciation, tandis que les deux autres, l'histoire qui est le produit de l'invention, et le récit, qui est le produit de la disposition et de la narration, concernent le produit d'une activité.
} 